The Egyptian Journal of Hospital Medicine (October 2020) Vol. 81 (6), Page 2179-2183

\title{
A Randomized-Trial Study of Corneal Collagen Cross-Linking (CXL) in Progressive-Keratoconus (PK)
}

Hassan Shamselden Yousef

\author{
Department of Ophthalmology, Faculty of Medicine, Al-Azhar University, Assiut, Egypt \\ Corresponding Author: Hassan Shamselden Yousef, Phone: +201144090050; E-Mail: hassanetal@ yahoo.com
}

\begin{abstract}
Background: Keratoconus is a frequent, familiar, bilateral, non inflammatory, and gradually progressive corneal ectasia, which leads to corneal protrusion, irregular astigmatism, and decrease vision. Currently, the corneal cross-linking (CXL) treatment could attain vision improvement.

Aim: To investigate the topographic, refractive, and clinical-out-comes after six months CXL in gradually progressing keratoconic eyes.

Patients \& Methods: A follow-up, randomized, controlled-trial study was used. In this study, 30eyes with progressivekeratoconus (PK) were recruited in the study. Cross-linking was done and achieved by a solution of $0.1 \%$ riboflavin mixture with $20 \%$ dextran for 15 minutes before and during 30minutes time-period of ultraviolet A (UV-A) irradiation $\left(3 \mathrm{Mwlcm}{ }^{2}\right)$. The obtained data were analysed using IBM SPSS software.

Results: Statistically significant improvements were noticed in Sim k (at 6months), Kmin (at 6months), spherical power (at 6momths), cylinder power (at 6momths), spherical equivalent [SE] (at 6months), uncorrected visual acuity (UCVA) (at 6months) and best corrected visual acuity [BCVA] (at 6months) in trial group (15patients) when compared with the controls.

Conclusions: At 6months, hopeful, promising improvements were reported in Kmax, UCVA, and BCVA. So, long term studies are requested to survey the efficiency of CXL as well as the safety of the repeated procedures.

Keywords: Corneal collagen cross-linking; Riboflavin; Epithelium-off procedure; Topographics changes; Keratoconus.
\end{abstract}

\section{INTRODUCTION}

Keratoconus is a common, bilateral, noninflammatory, and gradually progressive corneal ectasia; leads to corneal protrusion, irregular astigmatism, and lowering vision ${ }^{(\mathbf{1 , 2})}$. Change in the collagen fibrils structural organization in the cornea is deemed as its fundamental features ${ }^{(3)}$. In the advanced phases, the stroma could break leading to acute hydrops, resulting in considerabe visual deteruations ${ }^{(4)}$. Diagnosis of keratoconus relies on the presence of two of the three standard criteria, which consists of the anterior or posterior surface of the corneal steeping, progressively rising of corneal stromal thinning, and corneal thickness alteration in the thinnest site ${ }^{(\mathbf{1 - 3 , 5})}$. Keratoconus is a popular indication for corneal transplantation surgery ${ }^{(6)}$. However, corneal cross-linking (CXL) is the process, which could abstain the keratoconus progression and as well the other corneal ectasia ${ }^{(7,8)}$.

In theory, CXL implentation in strengthing the corneal tissue is based on the concept that a proportional pacuity of covalent bonds in and between the collagen matrix that leads to a weakned biomechanical firmness of keratoconic cornea.

Therefore, it's supposed that the CXL treatment could encourage photochemically triggered cross-links in the collagen-network by using a mixture of vitamin B2 (riboflavin) and a long wave-length ultraviolet A
(UV-A) radiation $(370 \mathrm{~nm})$. Absorption of the topically applied riboflavin is generaly

obstructed because of the corneal epithelial tight junctions. Accordingly, the up-to-date gold-standard treatment protocols use the de-epithelialization to optimize the riboflavin permeation and improve its

absorption $^{(8)}$. However, reaction to riboflavin experience pharmacogenomic variation ${ }^{(7)}$, and variation in its efficiency is noticed in various settings ${ }^{(9)}$.

Over the past-decades, the CXL treatment could attain vision improvement ${ }^{(\mathbf{1 0}-\mathbf{1 2})}$, apical keratometry improvement, lowering the corneal curvature, SE refraction and refractive cylinder ${ }^{(\mathbf{1 3}, 14)}$ with boosting the contrast sensitivity (CS) ${ }^{(15,16)}$.

\section{AIM OF THE STUDY}

To investigate the topographic, refractive, and clinical-outcomes after six months CXL in gradually progressing keratoconic eyes.

\section{PATIENTS AND METHODS}

Study design and setting:

The present work was done using a prospective randomized-trial design, at a private eye center (the Future Eye Center, Sohag, Upper Egypt) on 30eyes of 15 patients with bilateral progressive-keratoconus (PK) as a study group and another 30 eyes of 15 patients with bilateral PK as a control group.

This article is an open access article distributed under the terms and conditions of the Creative Commons Attribution (CC BY-SA) license (http://creativecommons.org/licenses/by/4.0/) 


\section{Ethical considerations:}

A written informed agreement was obtained from all participants before enrollment in the study. The Ethics Committee of the Faculty of Medicine, Al-Azhar University approved the study.

\section{Measurements and diagnosis:}

In the current study, the maximum simulated keratometry $\left(\mathrm{K}_{\max }\right)$ value of the steepest axis on corneal topography was deemed as the basic out-come measurement. Further, UCVA, BCVA, spherical and cylindrical error on subjective refraction, $\mathrm{SE}$, the minimum simulated keratometry value, and the corneal thickness at the thinnest point were evaluated as secondary out-come measures.

Diagnosis of PK was deemed if there was subjective lowering of vision beside; at least 1 of the following 3 criteria; (1) An rise of at least 1.0D (diopter) in the steepest simulated keratometry value as assured by computerized videokeratography or in the steepest meridian assessed by manual keratometry, (2) An increase in astigmatism of at least 1.0D, and (3) A $0.1 \mathrm{~mm}$ or further lowering in back-optic-zone-radius of the best fitting contact-lens.

\section{Inclusion criteria:}

In the present study, all the patients were in the age range from $15-40 y e a r s$ with more than $400 \mu \mathrm{m}$ of minimum central corneal thickness (CCT) and topographic documentation of PK with no prior history of ocular diseases or ocular surgery.

\section{Exclusion criteria:}

Patients are excluded from this study if their ages were out the accepted age range, refused to give their written consent, have $\mathrm{K}_{\max }$ of more than $60 \mathrm{D}$, have CCT of less than $400 \mu \mathrm{m}$, as well as suffer from ocular surface disorders, apical corneal scaring, previous ocular surgery, hydrops, previous history of ocular herpes or active corneal ulcers, history of any associated autoimmune diseases, chemical or thermal burns, and diabetes. Lactating and/or pregnant women were also precluded from the study.

\section{Patients and controls:}

The included 30 patients were separated into two groups; the first is a control group incorporating 30 eyes that were given artificial tear drops only without CXL. The second is the trial group incorporating 30 eyes that were subjected to a riboflavin-assisted UV-A irradiation collagen cross-linking.

\section{The surgical procedures:}

All surgeries in the present study were achieved by a single surgeon (the author) using the same epithelium-off technique (Dresden protocol) ${ }^{(17)}$.

Briefly, topical anesthetic eye drops (Benoxinate $0.4 \%$ ) were instilled 3 times followed by two drops of the topical fluoroquinolone antibiotic (Moxifloxacin, Vigamox Alcon). Under sterile surgical conditions, the patient was placed in a supine position with the cornea perpendicular to the pointed laser beam. The skin was dis-infected by $5 \%$ sol. of povidoneiodine. A circular area of $8 \mathrm{~mm}$ diameter, at the central cornea, was subjected to $20 \%$ ethyl alcohol using a corneal trephine and the resultant swelled corneal epithelium was removed with stick knife or microsponge. Subsequent to an efficient wash of corneal surface, riboflavin $0.1 \%$, containing $20 \%$ dextran, was applied to the eye every 3-5minutes for 30 minutes. Using a UV-X lamp, UV-A irradiation, with a wave-length of $370 \mathrm{~nm}$, was then applied for 30 minutes from $50 \mathrm{~mm}$ from the apex of the cornea with an irradiance of $3 \mathrm{Mw} / \mathrm{cm}^{2}$.

During the UV-A exposure, the riboflavin application was continued every 4minutes and its adequate penetration was assured using slit-lamp examination.

Post fulfilment of the irradiation proceedings, the eye was rinsed with a sterile solution followed by two drops of the topical fluoroquinolone antibiotic (Moxifloxacin, Vigamox Alcon) and subsequently, a soft bandage contact-lens was placed.

Application of the topical fluoroquinolone antibiotic (Moxifloxacin, Vigamox Alcon) was continued 4times daily for 1week after surgery and the contact-lens remained in place until epithelial closure was confirmed. Moreover, a topical steroid was prescribed for the upcoming 3 weeks followed by a stepwise tapering with non-steroidal anti-inflammatory eye drops for the next 2 weeks and a preservative-free artificial tear drops for 6months.

\section{Follow-up procedures:}

A post-operative examination was achieved to each case on the $1^{\text {st }}, 3^{\text {rd }}$, and $7^{\text {th }}$ days till epithelial healing with repeated visits on end of the $6^{\text {th }}$ months.

\section{Out-come measures:}

In the current study, the recorded data include corneal topography parameters $\left(\operatorname{Sim} \mathrm{K}, \mathrm{K}_{\max }, \mathrm{K}_{\min }\right.$ ), subjective refraction (spherical power, cylinder power, and SE), visual acuity, and CS. The $K_{\max }, K_{\min }$, and Sim $\mathrm{K}$ were evaluated by pentacam and the CS was measured by the Pelli-Robson CS chart. The visual acuity was evaluated by LOG MAR chart.

\section{Statistical analysis}

The obtained results were subjected to a master tabulation in the MS Excel spread-sheet and data were analyzed using IBM SPSS software version 22.0 for windows. Results were presented as means \pm standard deviations (SD). We utilized t-test to evaluate significance; the level significance was accepted when the $p$ value was $\leq 0.05$.

\section{RESULTS}

In the trial group, the UCVA was improved from the base-line of $1.0 \pm 0.21$ to decrease subsequently to $0.06 \pm 0.02$ on end of the $6^{\text {th }}$ month (table 1 ). 
The BCVA in log MAR was found to be increased from its base-line value of $0.70 \pm 0.24$ to $0.3 \pm 0.12=$ in the $6^{\text {th }}$ month in the controls (table 2). In the trial group, the mean BCVA was raised from the base-line to $0.28 \pm 0.04$ and decreased to reach $0.06 \pm 0.01$ in end of the $6^{\text {th }}$ month (table $\mathbf{1}$ ).

The obtained findings of the current investigation revealed that the UCVA was improved in 7eyes from the base-line of the value of $1.0 \pm 0.54$ to $0.5 \pm 0.25$ on end of the six month in the control group (table 2 ).

Table (1): Comparison between base-line and last follow-up on end of the $6^{\text {th }}$ month clinical characteristics of 30 keratoconic eyes after CXL

\begin{tabular}{|l|c|c|c|}
\hline Parameter & $\begin{array}{c}\text { Before } \\
\text { CXL (n=30) } \\
(\mathbf{m e a n} \pm \text { SD) }\end{array}$ & $\begin{array}{c}\text { Last follow- } \\
\text { up after } \\
\text { CXL (n=30) } \\
(\mathbf{m e a n} \pm \text { SD) }\end{array}$ & $\begin{array}{c}\text { p- } \\
\text { value }\end{array}$ \\
\hline UCVA & $1.00 \pm 0.21$ & $0.06 \pm 0.02$ & 0.0007 \\
\hline BCVA & $0.28 \pm 0.04$ & $0.06 \pm 0.01$ & 0.003 \\
\hline $\mathbf{K}_{\text {min }}$ & $43.81 \pm 2.60$ & $43.18 \pm 2.50$ & 0.75 \\
\hline $\mathbf{K}_{\text {max }}$ & $48.05 \pm 2.75$ & $46.93 \pm 3.14$ & 0.225 \\
\hline $\mathbf{K}_{\text {mean }}$ & $45.18 \pm 1.36$ & $44.60 \pm 1.37$ & 0.37 \\
\hline Sphere & $-1.52 \pm 2.22$ & $-1.00 \pm 1.02$ & 0.03 \\
\hline Cylinder & $-2.82 \pm 0.80$ & $-1.68 \pm 1.00$ & 0.01 \\
\hline SE & $-3.75 \pm 0.74$ & $-1.75 \pm 1.00$ & 0.017 \\
\hline CS & $1.15 \pm 0.40$ & $1.12 \pm 0.40$ & 0.56 \\
\hline
\end{tabular}

BCVA: Best corrected visual acuity; CCT: Central corneal thickness; CS: contrast sensitivity; Cylinder: Cylinder reference error; $\mathbf{K}_{\text {max: }}$ Maximum keratometric value; $\mathbf{K}_{\text {mean: }}$ Mean keratometric value; $\mathbf{K}_{\mathbf{m i n}}$ Minimum keratometric value; SE: Spherical equivalent; UCVA: Uncorrected visual acuity.
Table (2): Comparison between base-line and last follow-up on end of the $6^{\text {th }}$ month clinical characteristics of 30 control eyes

\begin{tabular}{|l|c|c|c|}
\hline Parameter & $\begin{array}{c}\text { First visit } \\
(\mathbf{n = 3 0}) \\
(\mathbf{m e a n} \pm \\
\text { SD) }\end{array}$ & $\begin{array}{c}\text { Last } \\
\text { follow-up } \\
\text { after CXL } \\
(\mathbf{n = 3 0 )} \\
(\mathbf{m e a n} \pm \\
\text { SD) }\end{array}$ & $\begin{array}{c}\text { p- } \\
\text { value }\end{array}$ \\
\hline UCVA & $1.00 \pm 0.54$ & $0.50 \pm 0.25$ & 0.02 \\
\hline BCVA & $0.70 \pm 0.24$ & $0.30 \pm 0.12$ & 0.03 \\
\hline $\mathbf{K}_{\min }$ & $44.50 \pm 2.71$ & $45.60 \pm 3.02$ & 0.415 \\
\hline $\mathbf{K}_{\text {max }}$ & $47.01 \pm 2.82$ & $45.25 \pm 3.23$ & 0.05 \\
\hline $\mathbf{K}_{\text {mean }}$ & $45.02 \pm 1.55$ & $46.02 \pm 1.84$ & 0.247 \\
\hline Sphere & $-1.45 \pm 2.34$ & $-2.32 \pm 2.55$ & 0.71 \\
\hline Cylinder & $-2.88 \pm 0.92$ & $-3.44 \pm 0.88$ & 0.001 \\
\hline SE & $-3.23 \pm 0.51$ & $-4.08 \pm 0.84$ & 0.02 \\
\hline CS & $1.16 \pm 0.46$ & $0.90 \pm 0.30$ & 0.04 \\
\hline
\end{tabular}

BCVA: Best corrected visual acuity; CCT: Central corneal thickness; CS: contrast sensitivity; Cylinder: Cylinder reference error; $\mathbf{K}_{\text {max: }}$ Maximum keratometric value; $\mathbf{K}_{\text {mean: }}$ Mean keratometric value; $\mathbf{K}_{\text {min: }}$ Minimum keratometric value; SE: Spherical equivalent; UCVA: Uncorrected visual acuity.

No significant reductions were observed in the present study in the $\mathrm{K}_{\max }$ value in both the controls and the trial group. The mean $\mathrm{K}_{\min }$ value was gradually decreased from its base-line value to the ultimate follow-up step of 6 months in the trial group. In the control group, a gradual increase was recorded in the mean $K_{\min }$ value from the base-line to the 6 month follow-up, as depicted in Table $\mathbf{1} \boldsymbol{\&} \mathbf{2}$.

The mean Sim K value in the control group was increased from the base-line to the follow-up period of 6 months. In the trial group, the $\operatorname{Sim} \mathrm{K}$ value was decreased from the base-line to the follow-up period of 6months as described in Figure 1A-C). 


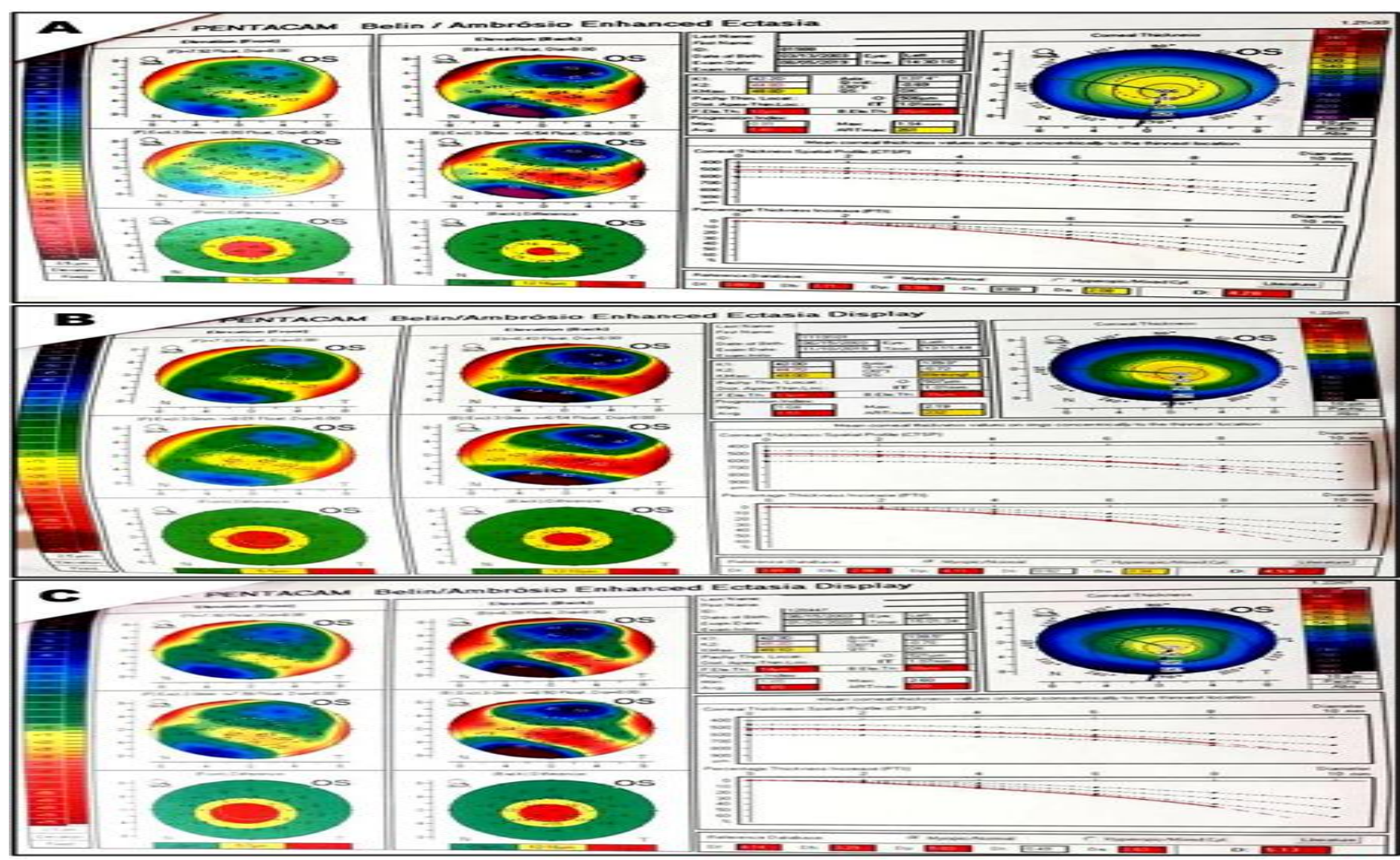

Figure (1): Pentacam picture of cases before treatment A and after treatment B and C

In the trial group, the mean spherical power was decreased from the base-line of $-1.52 \pm 2.22$ to $-1.00 \pm 1.02$ at the $6^{\text {th }}$ month, with a statistically significant decrease in spherical power at the $6^{\text {th }}$ months (table 1). The mean pre-operative spherical refractive error in the controlls was found to increase from $1.45 \pm 2.34$ to $-2.32 \pm 2.55$ at the $6^{\text {th }}$ month (table 2 ).

In the trial group, the mean cylinder power was found to decrease from $-2.82 \pm 0.80$ to $-1.68 \pm 1.00$ at the $6^{\text {th }}$ month (table 1 ). The mean cylinder power was increased in the control group from its base-line of -2.88 \pm 0.92 to $-3.44 \pm 0.88$ in the $6^{\text {th }}$ month (table 2 ).

In the trial group, SE was decreased from a base-line of $-3.75 \pm 0.74 \mathrm{D}$ to $-1.75 \pm 1.00 \mathrm{D}$ in the $6^{\text {th }}$ month, with a statistically significant decrease in the SE at the $6^{\text {th }}$ months (table 1). The mean SE was increased from $-3.23 \pm 0.51 \mathrm{D}$ to $-4.08 \pm 0.84 \mathrm{D}$ in the $6^{\text {th }}$ month in the control group (table 2).

In the trial group, the CS was decreased from $1.15 \pm 0.40 \log$ CS to increase subsequently to $1.12 \pm 0.40$ in the $6^{\text {th }}$ month (table 1). In the control group, the mean CS was decreased from $1.16 \pm 0.46 \log \mathrm{CS}$ to $0.90 \pm 0.30 \log$ CS in the $6^{\text {th }}$ month (table 2 ).

\section{DISCUSSION}

The CXL appears to display an essential role in halting the progression of keratoconus. Despite the widespread global reports of CXL, long term investigations are still requested. ${ }^{(18,19)}$. The gained results in the present study continue to demonstrate a statistically significant amelioration in the UCVA,

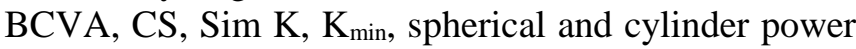
and $\mathrm{SE}$ in the trial group. In the $\mathrm{K}_{\max }$, the improvement in the trial group appeared to be statistically insignificant at a follow-up time of six months. In the controls; the $\operatorname{Sim~} \mathrm{K}, \mathrm{K}_{\max }$, and $\mathrm{K}_{\min }$ were increased and the UCVA, BCVA, spherical and cylinder power, SE, and CS were worsen designating advancement of the illness.

Additionally, the maximum central curvature, as measured by Sim K, gives an idea about corneal curvature of a central visually significant $3 \mathrm{~mm}$ area. It is known that the retrogression of $\operatorname{Sim} \mathrm{K}$ is a sign of treatment efficacy. A similar decrease in Sim K following CXL therapy was recorded by Viswanathan and his coworkers when they observed a remarkable reduction in Sim $\mathrm{K}$ value ${ }^{(\mathbf{2 0})}$. Therapy effectiveness is evaluated by $K_{\min }$ value, in the present research, in the trial group $\mathrm{K}_{\min }$ value decreased in all the follow-up periods, whereas it was increased in the control group from base-line. Similarly, Wittig-Silva et al. ${ }^{(21)}$ reported a significant difference between the trial and control groups. Despite of these reports, long-range studies still needed to explore how long this progresses and how long it is maintained.

The $\mathrm{K}_{\max }$ is a topographical mark for the effectiveness of riboflavin assisted CXL; it evaluates the seriousness of the illness advancement ${ }^{(\mathbf{1 1})}$. Many 
researches cleared a significant lowering in $\mathrm{K}_{\max }$ value after CXL therapy because of making flat effect on the cone by the CXL procedure ${ }^{(21,22)}$. The currently presented $\mathrm{K}_{\max }$ mean values were less than that of the controls at 3 and 6months, though this reduction was statistically insignificant. Advancement was obviously noticed among the controls, while it was arrested among the trial group. $\mathrm{K}_{\max }$ denotes the steepest anterior corneal curvature, is used to emphasize ectatic progression, and is a sign for CXL efficiency.

\section{CONCLUSION}

This study cleared that CXL is believed to be considered as an optimistic first line therapy option for almost all patients with PK. Particularly in view of our hopeful long term results, keratoconus is not a curable illness and its advancement is greatly changeable among different patients. The CXL has cleared efficiency in hindrance the illness advancement. The robustness of the stiffening impact of CXL is unclear.

Conflicts of interest: There are no conflicts of interest.

\section{Acknowledgement:}

The author is deeply thankful to the nursing staff in the Future Eye Center, Sohag, Upper Egypt for their assistance.

\section{REFERENCES}

1. Kennedy R, Bourne W, Dyer J (1986): A 48-year clinical and epidemiologic study of keratoconus. Am J Ophthalmol., 101:267-273.

2. Rabinowitz Y (1988): Keratoconus. Surv Ophthalmol., 42:297-319.

3. Krachmer J, Feder R, Belin M (1984): Keratoconus and related noninflammatory corneal thinning disorders. Surv Ophthalmol., 28:293-322.

4. Tao X, Yu H, Zhang Y, Li Z, Jhanji V, Ni S et al. (2013): Role of corneal epithelium in riboflavin/ultraviolet-A mediated corneal cross-linking treatment in rabbit eyes. Biomed Res Int., 2013:624563.

5. Gomes J, Tan D, Rapuano C, Belin M, Ambrosio R, Guell J et al. (2013): Global consensus on keratoconus and ectatic diseases. Cornea, 34:359-369.

6. Williams K, Muehlberg S, Lewis R, Coster D (1997): Long-term outcome in corneal allotransplantation. The Australian Corneal Graft Registry. Transplant Proc., 29 (12):983.

7. Cornelius N, Frerman F, Corydon T, Palmfeldt J, Bross P, Gregersen N et al. (2012): Molecular mechanisms of riboflavin responsiveness in patients with ETF-QO variations and multiple acyl-CoA dehydrogenation deficiency. Hum Mol Genet., 21:3435-3448.
8. Jankov-Ii M, Jovanovic V, Nikolic L, Lake JC, Kymionis G, Coskunseven E (2010): Corneal collagen cross-linking. Middle East Afr J Ophthalmol., 17:21-27.

9. Hutt F (1961): Genetic variation in the utilization of riboflavin, thiamine, and other nutrients. Ann N Y Acad Sci., 91:659-666.

10. Agrawal V (2009): Corneal collagen cross-linking with riboflavin and ultraviolet - a light for keratoconus: results in Indian eyes. Indian J Ophthalmol., 57:111-114.

11. Caporossi A, Mazzotta C, Baiocchi S, Caporossi $T$ (2010): Long-term results of riboflavin ultraviolet a corneal collagen cross-linking for keratoconus in Italy: the Siena eye cross study. Am J Ophthalmol., 149:585-593.

12. Vinciguerra $P$, Albe E, Trazza $S$, Rosetta $P$, Vinciguerra $\mathbf{R}$, Seiler $\mathbf{T}$ et al. (2009): Refractive, topographic, tomographic, and aberrometric analysis of keratoconic eyes undergoing corneal cross-linking. Ophthalmology, 116:369378.

13. Padmanabhan $P$, Radhakrishnan $A$, Venkataraman AP, Gupta N, Srinivasan B (2014): Corneal changes following collagen cross linking and simultaneous topography guided photoablation with collagen cross linking for keratoconus. Indian J Ophthalmol., 62:229-235.

14. Wittig-Silva $\mathrm{C}$, Chan $\mathrm{E}$, Islam $\mathrm{F}$, Wu T, Whiting $\mathrm{M}$, Snibson G (2014): A randomized, controlled trial of corneal collagen cross-linking in progressive keratoconus: threeyear results. Ophthalmology, 121:812-821.

15. Lamy R, Netto C, Reis R, Procopio B, Porco T, Stewart J et al. (2013): Effects of corneal cross-linking on contrast sensitivity, visual acuity, and corneal topography in patients with keratoconus. Cornea, 32:591-596.

16. Szczotka L, Barr J, Zadnik K (2001): A summary of the findings from the Collaborative Longitudinal Evaluation of Keratoconus (CLEK) Study. CLEK Study Group. Optometry, 72:574-584.

17. Spoerl E, Hoyer A, Pillunat L, Raiskup F (2011): Corneal cross-linking and safety issues. Open Ophthalmol J., 5:14-6.

18. O'Brart D, Patel P, Lascaratos G, Wagh V, Tam C, Lee J et al. (2015): Corneal Cross-linking to Halt the Progression of Keratoconus and Corneal Ectasia: Seven-Year Follow-up. Am J Ophthalmol., 160:1154-1163.

19. Saffarian L, Khakshoor H, Zarei-Ghanavati M, Esmaily H (2010): Corneal Crosslinking for Keratoconus in Iranian Patients: Outcomes at 1 year following treatment. Middle East Afr J Ophthalmol., 17:365-368.

20. Viswanathan D, Kumar N, Males J (2014): Outcome of corneal collagen crosslinking for progressive keratoconus in paediatric patients. Biomed Res Int., 2014:140461.

21. Wittig-Silva $C$, Whiting M, Lamoureux $E$, Lindsay $R$, Sullivan L, Snibson G (2008): A randomized controlled trial of corneal collagen cross-linking in progressive keratoconus: preliminary results. J Refract Surg., 24:S720S725.

22. Wollensak G (2006): Crosslinking treatment of progressive keratoconus: new hope. Curr Opin Ophthalmol., 17:356-360. 